\title{
Uso de benzodiazepínicos em idosos no Brasil
}

\section{Use of benzodiazepines in the elderly in Brazil \\ Uso de benzodiazepinas en ancianos en Brasil}

Ana Karoline de Almeida Mendes ORCID: https://orcid.org/0000-0003-2654-7859 Universidade Ceuma, Brasil

E-mail: anakarolinemendesal@hotmail.com Izabely Lima Assunção ORCID: https://orcid.org/0000-0001-8262-9192 Universidade Ceuma, Brasil

E-mail: izabelyyyyy@gmail.com

Giuliana Maria Morais Gonzalez ORCID: https://orcid.org/0000-0003-1864-9310 Universidade Ceuma, Brasil

E-mail: giulianagonzalez0698@gmail.com

Victoria Alves do Nascimento ORCID: https://orcid.org/0000-0002-3127-7518 Universidade Ceuma, Brasil

E-mail: metodologiacientifica2@gmail.com Luciana Sousa Silva

ORCID: https://orcid.org/0000-0001-9961-7518 Universidade Ceuma, Brasil

E-mail: lucianaasousa@gmail.com

Daniel Geovane Silva Souza

ORCID: https://orcid.org/0000-0001-9423-311X Universidade Ceuma, Brasil

E-mail: Daniel.giovane.sousa@gmail.com

Lethícia Maria Morais Gonzalez

ORCID: https://orcid.org/0000-0002-0840-542X Universidade Ceuma, Brasil

E-mail: lethiciagonzales@ hotmail.com Yvilla Cristina Costa

ORCID: https://orcid.org/0000-0003-0192-6318 Universidade Ceuma, Brasil

E-mail: metodologiacientifica2@gmail.com

Gabriel Machado Chiacchio ORCID: https://orcid.org/0000-0002-6112-9057 Centro Universitário UNITPAC, Brasi

E-mail: metodologiacientifica2@gmail.com

Maria Caroline Leal Borges de Araújo ORCID: https://orcid.org/0000-0003-2549-5233

Centro Universitário UNITPAC, Brasil

E-mail:metodologiacientifica2@gmail.com

Thassia Almeida Matos Viana ORCID: https://orcid.org/0000-0003-3996-7078 Universidade Ceuma, Brasil

E-mail: metodologiacientifica2@gmail.com Bruna Martins Pereira ORCID: https://orcid.org/0000-0003-2291-2871 Universidade Ceuma, Brasil E-mail: bru_martt@hotmail.com Bruna Brito Feitosa

ORCID: https://orcid.org/0000-0001-6435-0665 Universidade Ceuma, Brasil E-mail: bdiasfeetosa@gmail.com Luan Carvalho Rosa ORCID: https://orcid.org/0000-0001-9354-7790 Faculdade de Medicina de Campos, Brasil E-mail: luancrosaa@outlook.com Samantha cunha Vieira ORCID: https://orcid.org/0000-0002-8831-2855 Universidade Ceuma, Brasil E-mail: samanthacvieira@gmail.com 


\section{Resumo}

Durante o processo de envelhecimento decorre de alterações fisiológicas no sistema nervoso e endócrino que propiciam o aparecimento da insônia, estima-se que os transtornos do sono afetam em torno de $50 \%$ dos idosos. O tratamento de primeira linha para a insônia são os benzodiazepínicos, está classe deve ser evitada pelos idosos, pois aumentam os riscos de queda. Este estudo trata-se de uma revisão bibliográfica sistemática de natureza quantitativa, que utilizou as plataformas PubMed, Scientific Eletronic Library On-line (SciELO) e Google Scholar como base de dados para pesquisa dos artigos científicos, publicados entre 2016 - 2021, nas línguas portuguesa e inglesa. Dos 200 resultados obtidos, 189 foram excluídos, restando apenas 11 artigos. Entre os artigos analisados, pôde-se observar que mesmo contraindicados para esta faixa etária, ainda é alta a taxa de uso de benzodiazepínicos por idosos no Brasil, sendo sua principal indicação atualmente o controle da insônia. Além disso, foi identificado haver fortes indícios de relação entre o uso prolongado dos BZD e o desenvolvimento de demências a longo prazo. Como há conhecimento 
dos efeitos colaterais por parte da população médica, esta deve instruir os pacientes sobre os riscos e evitar a iatrogenia.

Palavras-chave: Benzodiazepínicos; Idoso; Insônia.

\begin{abstract}
During the aging process results from physiological changes in the nervous and endocrine system that propitiate the onset of insomnia, it is estimated that sleep disorders affect around 50\% of the elderly. The first-line treatment for insomnia are benzodiazepines, this class should be avoided by the elderly because they increase the risk of fall. This study is a systematic bibliographic review of quantitative nature, which used the pubmed, scientific electronic library online (SciELO) and Google Scholar platforms as a database for the research of scientific articles, published between 2016 and 2021, in Portuguese and English. Of the 200 results obtained, 189 were excluded, leaving only 11 articles. Among the articles analyzed, it was observed that even if contraindicated for this age group, the rate of benzodiazepine use by the elderly in Brazil is still high, and its main indication is currently the control of insomnia. In addition, there were strong indications of a relationship between prolonged bzd use and the development of dementia in the long term. As there is knowledge of side effects by the medical population, this should instruct patients on the risks and avoid iatrogenic.
\end{abstract}

Keywords: Benzodiazepines; Elderly; Insomnia.

\title{
Resumen
}

Durante el proceso de envejecimiento como resultado de los cambios fisiológicos en el sistema nervioso y endocrino que propician la aparición del insomnio, se estima que los trastornos del sueño afectan a alrededor del 50\% de los ancianos. El tratamiento de primera línea para el insomnio son las benzodiazepinas, esta clase debe ser evitada por los ancianos porque aumentan el riesgo de caídas. Este estudio es una revisión bibliográfica sistemática de carácter cuantitativo, que utilizó las plataformas pubmed, scientific electronic library online (SciELO) y Google Scholar como base de datos para la investigación de artículos científicos, publicada entre 2016 y 2021, en portugués e inglés. De los 200 resultados obtenidos, se excluyeron 189, quedando sólo 11 artículos. Entre los artículos analizados, se observó que incluso si está contraindicado para este grupo de edad, la tasa de uso de benzodiazepinas por parte de los ancianos en Brasil sigue siendo alta, y su principal indicación es actualmente el control del insomnio. Además, hubo fuertes indicios de una relación entre el uso prolongado de bzd y el desarrollo de demencia a largo plazo. Como hay conocimiento de los efectos secundarios por parte de la población médica, esto debe instruir a los pacientes sobre los riesgos y evitar los iatrogénicos.

Palabras clave: Benzodiazepinas; Ancianos; Insomnio.

\section{Introdução}

O termo idoso para a Organização Mundial de Saúde (OMS) é utilizado para toda pessoa que tem 60 anos ou mais de idade, e essa mesma definição está presente na Política Nacional do Idoso de 1994 (estabelecida lei federal 8.842) e no Estatuto do Idoso de 2003 (lei 10.741) (de Freitas, de Souza, \& Coutinho, 2021).

Já o termo envelhecimento corresponde a um processo de desenvolvimento humano normal, por qual todo indivíduo passa, e ele consiste em alterações neurobiológicas estruturais, funcionais e químicas, como, por exemplo, defeitos nos núcleos da base, sinapses diminuídas e alteração na circuitaria colinérgica, respectivamente (Santos, Heidemann, Marçal, \& ArakawaBelaunde, 2019).

O envelhecimento, apesar de universal, acontece de formas diferentes em cada indivíduo. As alterações fisiológicas que todo ser humano apresenta quando chega a velhice, como perda auditiva e alterações de memória, são chamadas senescência. Porém, alguns idosos cursam com patologias que são típicos da velhice, por exemplo, doenças do coração, câncer, alzheimer, entre outros. Esse processo patológico é chamado senilidade (Miguel, 2021).

O processo de envelhecimento decorre de alterações fisiológicas em sistemas fundamentais como o imunológico, nervoso e neuroendócrino, contudo, esse comprometimento propicia o aparecimento e a instalação de doenças psicossomáticas. O envelhecimento bem-sucedido é onde os idosos almejam um estilo de vida saudável, como a prática de exercícios físicos, boa alimentação, o hábito de não fumar, entre outros, obtendo uma melhor e maior expectativa de vida. Todavia, por mais que a pessoa na terceira idade adquira um estilo de vida saudável existem certos danos biológicos que não podem ser reparados (Borges, 2017; Macena, Hermano, \& Costa, 2018). 
O sono é restaurador é fundamental para o correto funcionamento do organismo. Entretanto, o risco de desordens do sono aumenta com a idade, estima-se que os transtornos do sono afetam em torno de 50\% dos idosos (Pacini, 2020). Dentre essas desordens, insônia é a mais prevalente. Insônia é definida como uma dificuldade para iniciar o sono ou para se manter dormindo, quando pode haver uma diminuição total ou parcial da quantidade e/ou da qualidade do sono (Sá et al., 2019).

Diversos fatores podem ser a causa para a insônia, apneia do sono, síndrome das pernas inquietas e até mesmo efeito colateral de medicações, como anti-hipertensivos, anticolinérgicos, broncodilatadores, xantinas e antidepressivos inibidores da recaptação da serotonina. A insônia também pode estar relacionada ao uso de álcool, nicotina, cafeína e doenças, dentre elas destacam-se as patologias psiquiátricas, como ansiedade e depressão (Iroldi et al., 2020; Moreno et al., 2019).

Para tratamento da insônia são usados geralmente os anti-histamínicos e diazepínicos, porém muitas vezes estes podem causar efeito paradoxal em idosos, gerando a perda de sono. Outras terapias podem ser utilizadas como alternativa, como exemplo a higiene do sono, antidepressivos e agonistas de melatonina (Lopes et al., 2021; Pinto, 2018).

Os benzodiazepínicos potencializam o efeito do neurotransmissor ácido gama-aminobutírico (GABA) no receptor $\mathrm{GABA}_{\mathrm{A}}$, o que resulta em propriedades hipnóticas. O ácido gama-aminobutírico (GABA) é o principal neurotransmissor inibitório presente no sistema nervoso central, ele atua facilitando a entrada do cloreto, o que aumenta a diferença de potencial (DDP) da bomba de sódio e potássio, dificultando a despolarização e assim a passagem do impulso nervoso (Roberti, 2017).

No caso de idosos este efeito induz a tolerância e dependência e aumenta o risco de quedas. Idosos que sofrem de insônia têm uma probabilidade quatro vezes maior de sofrer uma queda, a qual pode resultar em ferimentos e fraturas (Fiorelli et al., 2017; Oliveira et al., 2020; Roberti, 2017).

\section{Metodologia}

Os procedimentos metodológicos de um trabalho acadêmico é um processo lógico com finalidade principal de atingir um determinado fim ou para se chegar ao conhecimento de um determinado assunto (Conforto, Amaral, \& Silva, 2011). Com isso, o seguinte trabalho de uma revisão bibliográfica sistemática de natureza quantitativa, que utilizou as plataformas PubMed, Scientific Eletronic Library On-line (SciELO) e Google Scholar como base de dados para pesquisa dos artigos científicos.

Foram utilizados os seguintes descritores de ciências em saúde (DeCS): idoso, receptores de benzodiazepínicos; insônia.

Os critérios de busca foram restritos a publicação dos últimos 5 anos. Os critérios de inclusão considerados foram: estudos envolvendo pacientes idosos 60 anos ou mais, de ambos os sexos, que abordassem a relação entre o uso de benzodiazepínicos em idosos no Brasil, prevalência e fatores de risco associados.

Foram utilizadas literaturas publicadas entre 2016-2021, nas línguas portuguesa e inglesa, que abordavam o seguinte tema: "Análise da relação entre do uso de benzodiazepínicos em idosos no Brasil".

Nesta revisão, os critérios de exclusão utilizados foram: Livros, documentos de projetos de dissertação, resumos em eventos, editoriais, relatos de caso isolados, artigos que não cumpriam os critérios de inclusão e artigos duplicados.

A escolha dos artigos a serem utilizados nesta revisão foi realizada por meio da leitura do título, resumo e, por fim, da leitura do artigo na íntegra, sendo realizada uma análise criteriosa dos artigos fundamentados nos critérios de inclusão e exclusão supracitados. De acordo com o mecanismo de busca, foram encontrados resultados, sendo eles na base de dados PubMed e Google Scholar, com na base SciELO (Quadro 1).

Dessa forma, utilizando- se os critérios de inclusão e exclusão supracitados, a metodologia desta pesquisa pode ser realizada de forma a alcançar resultados efetivos e reduzir possíveis erros, tornando esta revisão mais rigorosa cientificamente (Conforto et al., 2011). 


\section{Resultados e Discussão}

Dos 200 resultados obtidos, 189 foram excluídos com base nos critérios de exclusão para a pesquisa e, além disso, não abordavam de forma objetiva sobre o tema, sendo apenas 11 artigos utilizados na confecção do estudo. Todos os artigos escolhidos serão expostos no Quadro 1, seguindo a ordem de ano de sua publicação.

Quadro 1 - Artigos selecionados para pesquisa.

\begin{tabular}{|c|c|c|}
\hline TÍTULO & ANO, AUTOR & OBSERVAÇÕES \\
\hline $\begin{array}{l}\text { Association between Development of } \\
\text { Dementia and Use of Benzodiazepines: } \\
\text { A Systematic Review and Meta-Analysis }\end{array}$ & $\begin{array}{l}\text { (Lucchetta, da Mata, \& } \\
\text { Mastroianni, 2018) }\end{array}$ & $\begin{array}{l}\text { O estudo sugere uma associação entre o uso de } \\
\text { benzodiazepínicos e o desenvolvimento de demência. Os } \\
\text { resultados da meta-análise sugerem que os } \\
\text { benzodiazepínicos podem ser um fator de risco para o } \\
\text { desenvolvimento de demência. }\end{array}$ \\
\hline $\begin{array}{l}\text { The use of benzodiazepines by women } \\
\text { cared for at a Family Health Unit }\end{array}$ & $\begin{array}{l}\text { (Silva, Almeida, \& } \\
\text { 2019) }\end{array}$ & $\begin{array}{l}\text { O estudo sugere que o grupo que deve receber maior } \\
\text { atenção em relação ao consumo de benzodiazepínicos é } \\
\text { o de mulheres de meia-idade ou mais, com baixa } \\
\text { escolaridade e doenças crônicas. }\end{array}$ \\
\hline $\begin{array}{l}\text { Benzodiazepine deprescription strategies } \\
\text { in chronic users: a systematic review }\end{array}$ & (Ribeiro \& Schlindwein, 2021) & $\begin{array}{l}\text { O uso de benzodiazepínicos por um longo prazo tem } \\
\text { efeitos adversos frequentes, especialmente em adultos } \\
\text { idosos. Devido ao potencial de causar dependência, } \\
\text { baixa adesão do paciente e falta de consciência dos } \\
\text { efeitos colaterais. }\end{array}$ \\
\hline $\begin{array}{l}\text { Uso de psicofármacos entre idosos } \\
\text { residentes em comunidade: prevalência e } \\
\text { fatores associados }\end{array}$ & $\begin{array}{l}\text { (Abi-Ackel, Lima-Costa, } \\
\text { Castro-Costa, \& Loyola Filho, } \\
\text { 2017) }\end{array}$ & $\begin{array}{l}\text { A prevalência de uso de psicofármacos foi de } 13,4 \% \text {, } \\
\text { sendo } 8,3 \% \text { para uso de benzodiazepínicos e } 5,0 \% \text { para } \\
\text { antidepressivos. Os fatores independentemente } \\
\text { associados ao uso de psicofármacos foram sexo } \\
\text { feminino, relato de diagnóstico médico para depressão, } \\
\text { ter realizado } 5 \text { ou mais consultas médicas nos últimos } 12 \\
\text { meses. }\end{array}$ \\
\hline $\begin{array}{l}\text { Prevalência e fatores associados ao uso } \\
\text { de benzodiazepínicos em idosos da } \\
\text { comunidade }\end{array}$ & $\begin{array}{l}\text { (Alvim, Cruz, Vieira, Bastos, \& } \\
\text { Leite, 2017) }\end{array}$ & $\begin{array}{l}\text { Dentre os usuários de benzodiazepínicos, } 38,4 \% \text { também } \\
\text { utilizavam antidepressivos. O uso de desses fármacos se } \\
\text { mostrou associado à presença de transtornos mentais e } \\
\text { comportamentais autorrelatados, polifarmácia e } \\
\text { realização de consulta médica nos últimos três meses. }\end{array}$ \\
\hline $\begin{array}{l}\text { Caracterização das condições de saúde e } \\
\text { presença de polifarmácia em idosos } \\
\text { institucionalizados }\end{array}$ & (Gatto, 2017) & $\begin{array}{l}\text { Houve associação significativa entre o uso de } \\
\text { benzodiazepínicos e acidente vascular encefálico e } \\
\text { demência. }\end{array}$ \\
\hline $\begin{array}{l}\text { Análise Do Uso De Benzodiazepínicos } \\
\text { Por Idosos No Brasil: Uma Revisão } \\
\text { Sistemática Rápida }\end{array}$ & (Nogueira \& Costa, 2021) & $\begin{array}{l}\text { Os fatores predisponentes ao uso de BZD e } \\
\text { comorbidades que precipitam o uso de BZD são } \\
\text { angústia, situações de estresse, dificuldades para } \\
\text { enfrentar os problemas da vida cotidiana, falta de ânimo } \\
\text { e motivação na vida, transtorno de personalidade e } \\
\text { falecimento de familiares, ansiedade, depressão e } \\
\text { insônia. }\end{array}$ \\
\hline $\begin{array}{l}\text { Analysis of the effect of long-term ue of } \\
\text { benzodiazepine by the elderly: a } \\
\text { systematic literature review }\end{array}$ & (Cardoso et al., 2021) & $\begin{array}{l}\text { O estudo das bibliografias selecionadas mostrou que o } \\
\text { uso de benzodiazepínicos entre idosos tem alta } \\
\text { prevalência, exacerbando uma série de eventos adversos. }\end{array}$ \\
\hline
\end{tabular}

Fonte: Autores (2021). 
Entre os artigos analisados, pôde-se observar que ainda é alta a taxa de uso de benzodiazepínicos por idosos no Brasil, sendo sua principal indicação nos dias atuais o controle da insônia (Silva et al., 2021).

De acordo com a literatura, os transtornos do sono no idoso envolvem uma série de fatores que variam desde alterações no padrão de sono, como a diminuição da amplitude do ritmo circadiano sono-vigília, diminuição do tempo de latência para o sono REM e predomínio das fases N1 e N2 do sono nREM, até causas que provocam alterações na arquitetura do próprio sono, como a privação crônica do sono, diminuição da atividade física, redução da exposição à luz solar, mudanças da temperatura e hormônios e uso de fármacos (Geib, Cataldo Neto, Wainberg, \& Nunes, 2003; Rios, Peixoto, \& Senra, 2008). Essas alterações provocam nos idosos o aumento dos despertares noturnos e cochilos durante o dia, gerando a insônia.

Há duas modalidades atualmente no tratamento da insônia crônica: farmacológica e não farmacológica. Dentre as técnicas não farmacológicas, a mais utilizada envolve a terapia cognitivo-comportamental (TCC), que consiste em técnicas combinadas de terapia cognitiva, de relaxamento e higiene do sono, sendo essa última uma intervenção educacional que envolve controle de estímulos (Oliveira, 2015). Em contrapartida, a terapia farmacológica envolve uma série de classes medicamentosas, nas quais se incluem desde benzodiazepínicos, até hipnóticos não benzodiazepínicos, antidepressivos, antihistamínicos e melatonina (Poyares et al., 2003).

Os benzodiazepínicos, de acordo com Bernik (1999), são as drogas mais utilizadas em todo o mundo. Segundo Fraser (1998), essas drogas começaram a ser utilizadas pela comunidade desde a década de 1960 devido as suas propriedades ansiolíticas, hipnóticas e miorrelaxantes, no entanto, foi a partir da década de 80 que pesquisas começaram a ser realizadas sobre os efeitos de seu uso indiscriminado em longo prazo.

Dentre os usuários crônicos dessa droga, pode-se citar especialmente mulheres de meia idade que buscam efeitos ansiolíticos, e idosos que buscam o efeito hipnótico da medicação (Silva et al., 2019). No Brasil, segundo Nogueira \& Costa (2021), os principais fatores predisponentes ao uso de BZD em idosos são: insônia, angústia, ansiedade, depressão, falecimento de familiares, e situações de estresse, o que justifica o seu uso crônico de forma excessiva por indivíduos nessa faixa etária.

O efeito ansiolítico dos benzodiazepínicos está relacionado com o sistema gabaminérgico do sistema límbico. O ácido gama-aminobutírico (GABA) é um neurotransmissor com função inibitória capaz de atenuar as reações serotoninérgicas responsáveis pela ansiedade. Assim, os BZD seriam agonistas deste sistema, agindo sobre os receptores gabaminérgicos e potencializando a ação do GABA (Poyares et al., 2003). Devido a sua ação, seus efeitos adversos mais comuns incluem sintomas depressivos do Sistema Nervoso Central, como: sonolência, incoordenação motora, ressaca, dificuldade de concentração, amnésia, confusão mental e delírios, tornando-se uma classe medicamentosa relativamente 'perigosa' para ser utilizada em idosos (Senra et al., 2021).

Dentre os estudos analisados, foi identificado que há fortes indícios de relação entre o uso prolongado dos BZD e o desenvolvimento de demências em longo prazo, além de comprometimentos cognitivos e psicomotores (Gatto, 2017; Lucchetta et al., 2018). Ademais, os efeitos colaterais que envolvem a depressão do SNC podem causar em idosos consequências bastante danosas, como tontura, zumbidos, maior risco de quedas e fraturas devido à incoordenação motora, piora da memória por conta da amnésia anterógrada provocada pelo uso da medicação, sonolência excessiva diurna, o que aumenta a insônia durante o período noturno, risco aumentado de acidentes e maior risco de iatrogenia devido à interação medicamentosa, uma vez que idosos geralmente usufrem da polifarmácia (Ribeiro et al., 2021; Rocha, 2014; Senra et al., 2021).

A incidência de quedas em indivíduos idosos é alta se comparada com indivíduos pertencentes a outras faixas etárias. Isso ocorre especialmente devido fatores de risco ambientais e internos relacionados ao declínio funcional e cognitivo. Doenças crônicas e uso de múltiplas medicações, no entanto, são fatores que tem importância fundamental na história e manejo 
do paciente (ROCHA, 2014). Dessa forma, por terem como característica a depressão do SNC, os BZD são uma classe medicamentosa que deve ser bastante discutida antes de ser prescrita para pacientes mais velhos.

A prevalência do uso de psicofármacos em idosos é alta devido aos inúmeros fatores emocionais que os envolvem, em especial o falecimento de seus parceiros, a diminuição do contato social com amigos, e a perda de energia e limitações para realizar atividades do dia a dia. Segundo pesquisa realizada por Abi-Ackel et al. (2017), 8,3\% dos pacientes usavam benzodiazepínicos, enquanto que 5\%, antidepressivos. Já em outra pesquisa realizada por Alvim et al. (2017), dentre os usuários de BZD, 38,4\% também faziam uso de antidepressivos, mostrando a polifarmácia que margeia o avançar da idade.

\section{Conclusão}

De acordo com as literaturas analisadas, tornou-se perceptível que o uso de benzodiazepínicos ainda é bastante prevalente na sociedade, especialmente por mulheres acima dos 50 anos e idosos. Suas propriedades ansiolíticas, hipnóticas e miorrelaxantes são bastante procuradas nesta faixa etária, visto que são muitos os casos relatados de insônia, ansiedade, depressão, angústia e situações de estresse, justificando seu uso crônico.

Por ter seu efeito ansiolítico relacionado especialmente ao sistema gabaminérgico, há inúmeros efeitos adversos que contraindicam a prescrição dessa classe medicamentosa para idosos, uma vez que deprimem o Sistema Nervoso Central e podem aumentar o risco de quedas e fraturas, sonolência, amnésia e confusão mental. Assim, é de fundamental importância que os profissionais de saúde, especialmente psiquiatras, geriatras e clínicos gerais auxiliem o paciente da melhor forma possível, uma vez que a iatrogenia é bastante prevalente nessa faixa etária e o risco de quedas é cada vez maior com o avançar da idade. Além disso, nota-se a necessidade de novas pesquisas sobre a temática, de forma que a equipe multidisciplinar esteja mais atualizada sobre os desafios da presença da iatrogenia na comunidade, especialmente em pacientes mais idosos.

\section{Referências}

Abi-Ackel, M. M., Lima-Costa, M. F., Castro-Costa, É., \& Loyola Filho, A. I. d. (2017). Uso de psicofármacos entre idosos residentes em comunidade: prevalência e fatores associados. Revista Brasileira de Epidemiologia, 20, 57-69.

Alvim, M. M., Cruz, D. T. d., Vieira, M. D T., Bastos, R. R., \& Leite, I. C. G. (2017). Prevalência e fatores associados ao uso de benzodiazepínicos em idosos da comunidade. Revista Brasileira de Geriatria e Gerontologia, 20, 463-473.

Borges, E. (2017). O envelhecimento populacional: um fenômeno mundial. O envelhecimento populacional um fenômeno, 17.

Cardoso, A. G. A., dos Santos, L. R., de Fátima Souza, A., de Figueiredo, B. Q., Nogueira, E. C., Brito, E. N. D., . . Fernandes, R. A. (2021). Análise do efeito do uso a longo prazo de benzodiazepínicos por idosos: uma revisão sistemática de literatura. Research, Society and Development, 10(12), e01101220022-e01101220022.

Conforto, E. C., Amaral, D. C., \& Silva, S. d. (2011). Roteiro para revisão bibliográfica sistemática: aplicação no desenvolvimento de produtos e gerenciamento de projetos. Trabalho apresentado, 8 .

Freitas, L. Z., de Souza, H. C., \& Coutinho, L. S. B. (2021). Atualizações sobre a relação entre insônia e hipertensão em idosos. Research, Society and Development, 10(8), e18710917276-e18710917276.

Fiorelli, K., \& Assini, F. L. (2017). A prescrição de benzodiazepínicos no Brasil: uma análise da literatura. ABCS Health Sciences, 42(1).

Gatto, C. M. (2017). Caracterização das condições de saúde e presença de polifarmácia em idosos institucionalizados.

Geib, L. T. C., Cataldo Neto, A., Wainberg, R., \& Nunes, M. L. (2003). Sono e envelhecimento. Revista de Psiquiatria do Rio Grande do Sul, 25, 453-465.

Iroldi, G. F., Alves, É. d. S., Luchesi, B. M., Cardoso, J. d. F. Z., Pavarini, S. C. I., \& Inouye, K. (2020). Associações entre estresse, sintomas depressivos e insônia em idosos. Jornal Brasileiro de Psiquiatria, 69, 228-238.

Lopes, J. M., Galvão, F. D., \& Oliveira, A. G. R. d. C. (2021). Risco de Morte em Idosos com Sonolência Excessiva Diurna, Insônia e Depressão: Estudo de Coorte Prospectiva em População Urbana no Nordeste Brasileiro. Arquivos brasileiros de cardiologia, 117, 446-454.

Lucchetta, R. C., da Mata, B. P. M., \& Mastroianni, P. d. C. (2018). Association between development of dementia and use of benzodiazepines: a systematic review and meta-analysis. Pharmacotherapy: The Journal of Human Pharmacology and Drug Therapy, 38(10), 1010-1020.

Macena, W. G., Hermano, L. O., \& Costa, T. C. (2018). Alterações fisiológicas decorrentes do envelhecimento. Revista Mosaicum(27), 223-238. 
Research, Society and Development, v. 11, n. 2, e32511225820, 2022

Miguel, M. B. (2021). Monoxí: kit de alerta para vazamento de monóxido de carbono.

Moreno, C. R. d. C., Santos, J. L. F., Lebrão, M. L., Ulhôa, M. A., \& Duarte, Y. A. d. O. (2019). Problemas de sono em idosos estão associados a sexo feminino, dor e incontinência urinária. Revista Brasileira de Epidemiologia, 21, e180018.

Nogueira, P. L. d. A., \& Costa, J. M. (2021). Análise do uso de benzodiazepínicos por idosos no Brasil: uma revisão sistemática rápida.

Oliveira, A. L. M. L., Nascimento, M. M. G. d., Castro-Costa, É., Firmo, J. O. A., Lima-Costa, M. F., \& Loyola Filho, A. I. d. (2020). Aumento da utilização de benzodiazepínicos entre idosos mais velhos: Projeto Bambuí. Revista Brasileira de Epidemiologia, 23, e200029.

Oliveira, S. P. L. d. (2015). Terapias não farmacológicas na insónia em idade avançada.

Pacini, E. (2020). Prevalência da insônia e fatores associados em adultos e idosos na atenção primária à saúde.

Pinto, R. d. O. A. (2018). Estratégias para diminuição do uso indiscriminado de benzodiazepínicos pelos pacientes do Programa de Saúde da Família do município de Dona Euzébia-Minas Gerais.

Poyares, D., Rizzo, G. N. V., Minhoto, G., Pinto Jr, L. R., de Souza Bezerra, M. L., Rodrigues, R. N. D., \& Tavares, S. M. A. (2003). I consenso brasileiro de insônia. CEP, 4020, 060 .

Ribeiro, P. R. d. S., \& Schlindwein, A. D. (2021). Benzodiazepine deprescription strategies in chronic users: a systematic review. Family Practice.

Rios, A. L. M., Peixoto, M. d. F. T., \& Senra, V. L. F. (2008). Transtornos do sono, qualidade de vida e tratamento psicológico. Núcleo de Estudos AC-PC. Universidade Vale do Rio Doce. Governador Valadares.

Roberti, F. (2017). Medicamentos Em Idosos Que Causam Prejuízo À Memória. Salão do Conhecimento.

Rocha, E. K. P. (2014). O uso crônico de Benzodiazepínicos na Saúde do idoso.

Sá, R. M. B. d., Motta, L. B. d., \& Oliveira, F. J. d. (2019). Insônia: prevalência e fatores de risco relacionados em população de idosos acompanhados em ambulatório. Revista Brasileira de Geriatria e Gerontologia, 10, 217-230.

Santos, P. A. d., Heidemann, I. T. S. B., Marçal, C. C. B., \& Arakawa-Belaunde, A. M. (2019). A percepção do idoso sobre a comunicação no processo de envelhecimento. Audiology-Communication Research, 24.

Senra, E. D., Queiroz, G. S., de Freitas Brito, Y., \& Camargo, M. R. (2021). Efeitos colaterais do uso crônico e indiscriminado de benzodiazepínicos: Uma revisão narrativa. Brazilian Journal of Development, 7(11), 102013-102027.

Silva, A. L. S., Dias, Í. K. B., de Paula Gusmão, L., \& Pinheiro, J. T. G. (2021). O uso de psicofármacos na população idosa: uma revisão integrativa da literatura. Bionorte, $10(\mathrm{~S} 2)$.

Silva, P. A. d., Almeida, L. Y. d., \& Souza, J. d. (2019). The use of benzodiazepines by women cared for at a Family Health Unit. Revista da Escola de Enfermagem da USP, 53. 